\title{
Aetiologies and Short-term Outcomes of Acute Kidney Injury in a Tertiary Centre in Southwest Nigeria
}

\author{
Adejumo Oluseyi ${ }^{1}$ Akinbodewa Ayodeji $^{1}$, Fasaanu Ayodeji ${ }^{1}$
}

\begin{abstract}
BACKGROUND: Acute kidney injury (AKI) has become a global health problem and is associated with increased morbidity, mortality and overall health expenditure. Information on the epidemiology and outcomes of AKI will help to audit practice and advocate for policies that will reduce this burden. This study determined aetiologies, short term outcomes and their predictors in AKI patients in a tertiary hospital in Southwest Nigeria.

METHODS: This was an 18-month retrospective study that involved 91 patients with AKI. The socio-demographic information, aetiology, severity and the treatment given to patients were recorded. Outcomes and their predictors were determined using multivariate analysis. P value $<0.05$ was taken as statistically significant.

RESULTS: The mean age of the study population was $45.12 \pm 20.67$ years. Common causes of AKI were sepsis in 50(54.9\%), hypovolaemia in 23(25.3\%), cardiac failure in 7(7.7\%) and eclampsia in 6(6.6\%). Fifty-seven (62.6\%) presented with stage 3. Thirty-one (34.1\%) had haemodialysis. Forty-eight (52.7\%) had complete renal recovery, 35(38.5\%) died and 3(3.3\%) left against medical advice while five (5.5\%) were referred to other hospitals. Stage 3 AKI (Adjusted odd ratio: 6.79, confidence interval: 1.21:38.04, $p=0.029$ ) and age $\geq 65$ years (Adjusted odd ratio: 4.14, confidence interval: 1.32-13.04, $p=0.015$ ) were significant predictors of mortality in AKI patients.

CONCLUSION: Sepsis and hypovolaemia were the commonest causes of AKI. The associated mortality is still high and factors associated with mortality were late presentation and older age. Early presentation, treatment and making haemodialysis affordable are key to improving AKI outcomes. KEYWORDS: aetiologies, outcomes, acute kidney injury
\end{abstract}

DOI:http://dx.doi.org/10.4314/ejhs.v26i1.8

\section{INTRODUCTION}

Acute kidney injury (AKI) has become a global health problem due to its increasing incidence in both developing and developed countries.(1,2). The true incidence of AKI is not known especially in developing countries due to poor data collection, underreporting and non-uniform criteria for diagnosis $(3,4)$. Some cases of AKI also go unrecognized while some patients with AKI do not present in the hospitals (5). The hospital-pooled incidence of AKI in a metanalysis that mainly involved studies from Asia, America and Europe in both adults and children was reported by Susantitaphong et al to be $22 \%$ and $14 \%$ respectively (2). The incidence of AKI ranges between $20-50 \%$ among patients admitted in the intensive care unit (6).

It is well established that the burden of AKI is quite enormous. It is associated with increased morbidity, mortality and overall health expenditure (7-9). Acute kidney injury has been reported to be an important risk factor for chronic kidney disease (CKD), rapid progression to end stage renal disease (ESRD) later in life and longterm non-renal morbidity and mortality $(10,11)$. Therefore, measures aimed at reducing the burden of AKI would also translate into reducing the burden of CKD which is beyond what most developing countries like Nigeria could cope with.

${ }^{1}$ Kidney Care Center, University of Medical Sciences, Ondo, Ondo State, Nigeria

Corresponding Author: Adejumo, Oluseyi, Email: ceeward2010@yahoo.com 
Collaborative efforts of government, international organizations, health care providers and corporate organizations are key to achieving the initiative of the International Society of Nephrology (ISN) of eliminating AKI by 2025. Data on the epidemiology and outcomes of AKI is required to enlighten the public to advocate for policies from the government and corporate organizations that will help reduce the burden of AKI. Determining the aetiologies and outcomes of AKI will also serve as audit of practice of health care workers with the aim of improving practice and reducing the burden of AKI. This will help in directing preventive strategies towards the common aetiologies of AKI.

The aim of this study was to determine the aetiologies, short term outcomes and their predictors in AKI patients in a tertiary health centre in Southwest Nigeria.

\section{METHODS}

This study was a retrospective study carried out in Kidney Care Centre, Ondo, Ondo state, Nigeria, which is a state-government owned tertiary hospital located in Southwest Nigeria and receives referrals from other departments within the hospital complex, other hospitals within and outside Ondo state, Nigeria. This study was a retrospective study that covered a period of 18 months between March 2014 and August 2015. Ethical clearance was obtained from ethical committee on research of the Ondo state hospital management board. Inclusion criteria were patients $\geq 15$ years of age presenting with AKI or who developed AKI while in the hospital. Patients with CKD or acute or chronic kidney disease were excluded.

Case notes and dialysis records of all patients who fulfilled the inclusion criteria were retrieved and reviewed. Data obtained from patients' records were age, gender, aetiology and stage of AKI at presentation, investigations done, estimated glomerular filtration rate (GFR) at presentation, treatment given, fluid input and output records, number of haemodialysis sessions for those who had renal replacement therapy (RRT) and outcomes following treatment. The estimated GFR was calculated using Modification of Diet in Renal Disease formula which has been validated in Nigerians (12). The possible outcomes in this study were left against medical advice, referral to another hospital, in-hospital mortality, recovery of renal function or dialysis dependence beyond 12 weeks.

Acute kidney injury was defined and staged using Kidney Disease Improving Global Outcome criteria (KDIGO) (13).Acute kidney injury was defined as increase in serum creatinine by 0.3 $\mathrm{mg} / \mathrm{dl}$ (26.5micromol/L) within 48 hours, or increase in serum creatinine to 1.5 times baseline, which is known or presumed to have occurred within the previous 7 days, or urine volume 0.5 $\mathrm{ml} / \mathrm{kg} / \mathrm{h}$ for 6 hours.

Stage 1: Serum creatinine rise of $\geq 26$ micromol/L within 48 hours or $50-99 \%$ rise from baseline within 7 days or urinary output of $<0.5 \mathrm{ml} / \mathrm{kg}$ per hour for more than 6 hours

Stage 2: 100-199\% rise in serum creatinine from baseline within 7 days or urinary output of $<0.5 \mathrm{mls} / \mathrm{kg}$ per hour for more than 12 hours

Stage 3: $\geq 200 \%$ rise in serum creatinine from baseline within 7 days or concentration of $\geq 354$ micromol/L within $48 \mathrm{hr}$ or $\geq 50 \%$ rise from baseline within 7 days or any requirement for RRT or urinary output of $<0.3 \mathrm{ml} / \mathrm{kg}$ per hour for 24 hours or anuria for 12 hours.

\section{Operational Definitions}

Renal recovery after AKI: Dialysis independence at hospital discharge (14)

Sepsis: Presence of a proven or suspected microbial infection in the presence of at least two of the following- temperature $>38^{\circ} \mathrm{C}$ or $<36^{\circ} \mathrm{C}$, pulse rate $>90 /$ minute, respiratory rate $>$ 24 cycles/minute, white cell count of $>$ 12,000 cells $/ \mathrm{mm}^{3}$ or $<4000$ cells $/ \mathrm{mm}^{3}$ (15)

Hypovolaemia: Significant fluid loss with features of dehydration and changes in the cardiovascular system such as tachycardia and hypotension

Surgical AKI: Acute Kidney Injury caused by surgical problems such as intestinal obstruction, enterocutaneous fistula, bone fractures or following surgical procedures

Obstetric/Gynaecologic: Acute Kidney Injury caused by obstetric or gynaecologic conditions such as eclampsia, abortion, ante-partum or postpartum haemorrhage and ectopic pregnancies

Medical AKI: Acute Kidney Injury caused by medical conditions such as gastroenteritis, heart 
failure or following medical treatment such as use of nephrotoxic medications

Data were analyzed using the statistical Package for Social Sciences (SPSS) version 17.0 by Chicago Inc. Results were presented in tabular form. Discrete variables were presented as frequency and percentages. Continuous variables were presented as mean and standard deviation for unskewed data and median, interquartile range for skewed data. Chi-square test was used to determine the significance of the observed differences for categorical variables while chisquare with trend was used where the categorical variable was ordinal. Binary logistic regression was used to determine the significant independent predictors of outcomes of AKI. P values $<0.05$ were considered statistically significant.

\section{RESULTS}

A total of 91 patients (50 males and 41 females) were involved in the study with a mean age of $45.12 \pm 20.67$ years. Sixty-nine $(75.8 \%)$ of these patients were less than 65 years. The median value and interquartile range of serum creatinine, urea and estimated GFR of these patients were 584.4(304.0)micromol/L, $19.6(15.0) \mathrm{mmol} / \mathrm{L}$ and $12.9(12.6) \mathrm{mls} / \mathrm{min} / 1.72 \mathrm{~m}^{2}$ respectively (Table 1).

The causes of AKI in this study were sepsis $50(54.9 \%)$, hypovolaemia 23(25.3\%), cardiac failure $7(7.7 \%)$ eclampsia $6(6.6 \%)$ and others $5(5.5 \%)$. Medical causes of AKI accounted for $53(58.2 \%)$, surgical causes for $20(22.0 \%)$ and obstetric and gynaecologic causes for the remaining 18(19.8\%) (Table 1).

Table 1: Characteristics of study population

\begin{tabular}{|c|c|}
\hline Parameter & $\begin{array}{l}\text { Frequency (percentages)/Mean (SD)/Median(IQR) } \\
\qquad(\mathrm{N}=91)\end{array}$ \\
\hline Mean age* (years) & $45.12(20.67)$ \\
\hline Median serum creatinine $\dagger($ micromol/L) & $584.4(304.0)$ \\
\hline Median serum urea $\dagger(\mathrm{mmol} / \mathrm{L})$ & 19.6(15.0) \\
\hline Median eGFR $†\left(\mathrm{mls} / \mathrm{min} / 1.72 \mathrm{~m}^{2}\right)$ & $12.9(12.6)$ \\
\hline Median HD sessions $\dagger$ & $3(2)$ \\
\hline \multicolumn{2}{|l|}{ Age groups } \\
\hline$\leq 65$ years & $69(75.8)$ \\
\hline$>65$ years & $22(24.2)$ \\
\hline \multicolumn{2}{|l|}{ Gender } \\
\hline Male & $51(45.1)$ \\
\hline Female & $40(54.9)$ \\
\hline \multicolumn{2}{|l|}{ Aetiology by specialty } \\
\hline Medical & $53(58.2)$ \\
\hline Obs\&Gyn & 18(19.8) \\
\hline Surgical & $20(22.0)$ \\
\hline \multicolumn{2}{|l|}{ Aetiology } \\
\hline Cardiac failure & $7(7.7)$ \\
\hline Eclampsia & $6(6.6)$ \\
\hline Sepsis & $50(54.9)$ \\
\hline Hypovolaemia & $23(25.3)$ \\
\hline Others & $5(5.5)$ \\
\hline \multicolumn{2}{|l|}{ KDIGO Stage of AKI } \\
\hline 1 & $12(13.2)$ \\
\hline 2 & $22(24.2)$ \\
\hline 3 & $57(62.6)$ \\
\hline \multicolumn{2}{|l|}{ Dialysis Required } \\
\hline Yes & $50(54.9)$ \\
\hline No & 41(47.1) \\
\hline \multicolumn{2}{|l|}{ Dialysed } \\
\hline Yes & $31(34.1)$ \\
\hline No & $60(65.9)$ \\
\hline
\end{tabular}

OBY\&GYN (obstetric and gynaecology), LAMA (left against medical advice), eGFR (estimated glomerular filtration rate), IQR (interquartile range), SD (standard deviation), *expressed as Mean (SD), †expressed as Median(IQR) 
Fifty-seven $(62.6 \%)$ of the patients presented with stage 3 AKI, 22(24.2\%) with stage 2 AKI and the remaining $12(13.2 \%)$ with stage 1 AKI. Thirtyone $(38.5 \%)$ of the patients were managed with haemodialysis with a median of 3 dialysis sessions.

The in-patient mortality rate of the patients in this study was $38.5 \%$ while $52.7 \%$ survived with complete renal recovery. Three patients $(3.3 \%)$ left against medical advice while five $(5.5 \%)$ were referred to other hospitals (Figure 1).

Fig 1: Outcomes of Patients with AKI

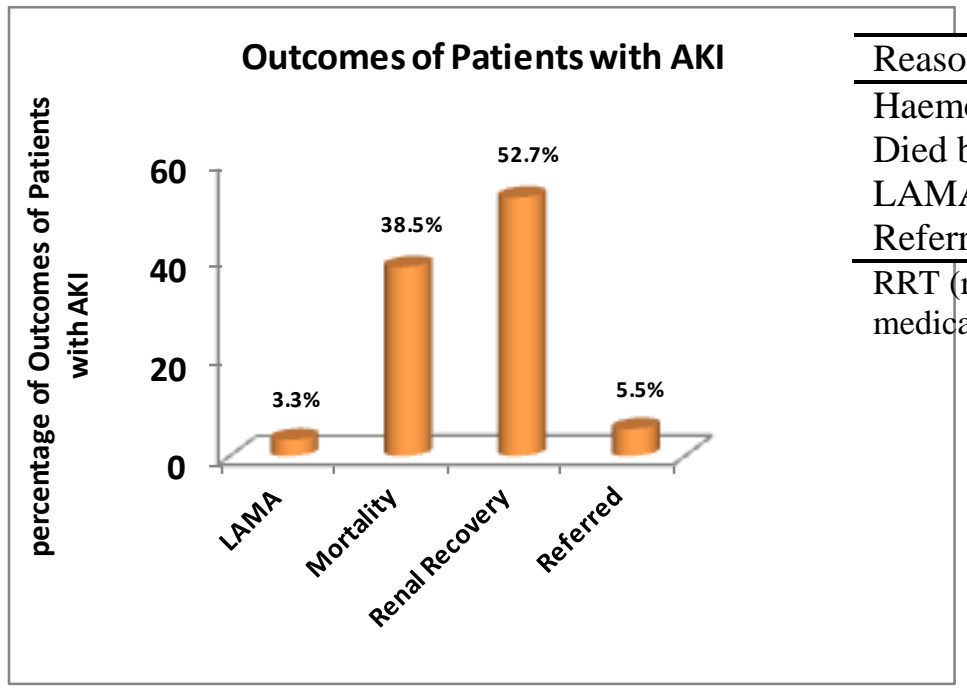

Table 3: Association between patients' characteristics and outcomes

\begin{tabular}{|c|c|c|c|c|c|}
\hline & $\begin{array}{c}\text { Death }(\mathrm{n}=35) \\
\mathrm{n}(\%)\end{array}$ & $\begin{array}{c}\text { Renal recovery }(\mathrm{n}=48) \\
\mathrm{n}(\%)\end{array}$ & OR & $95 \% \mathrm{CI}$ & $\mathrm{P}$ value \\
\hline \multicolumn{6}{|l|}{ Gender } \\
\hline Male & $18(40.0)$ & $27(60.0)$ & 0.82 & $0.34-1.97$ & 0.416 \\
\hline Female & $17(44.7)$ & $21(55.3)$ & & & \\
\hline \multicolumn{6}{|l|}{ Age } \\
\hline$<65$ years & $22(35.5)$ & $40(64.5)$ & 2.96 & $1.06-8.22$ & 0.034 \\
\hline$\geq 65$ years & $13(61.9)$ & $8(38.1)$ & & & \\
\hline \multicolumn{6}{|l|}{ AKI Staging } \\
\hline Stage 1 & $2(16.7)$ & $10(83.3)$ & 1 & & \\
\hline Stage 2 & $7(36.8)$ & $12(63.2)$ & 2.92 & $0.49-17.32$ & 0.046 \\
\hline Stage 3 & $26(50.0)$ & $26(50.0)$ & 5.00 & $0.99-25.08$ & \\
\hline \multicolumn{6}{|l|}{ Aetiology } \\
\hline Sepsis & $23(50.0)$ & $23(50.0)$ & & & \\
\hline Hypovolaemia & $6(30.0)$ & $14(70.0)$ & & & \\
\hline Cardiac Failure & $4(57.1)$ & $3(42.9)$ & & & 0.190 \\
\hline Eclampsia & $2(33.3)$ & $4(67.7)$ & & & \\
\hline Others & $0(0)$ & $4(100)$ & & & \\
\hline \multicolumn{6}{|l|}{ Specialty } \\
\hline Medical & $21(43.8)$ & $27(56.2)$ & & & \\
\hline OBGY & $7(41.2)$ & $10(59.8)$ & & & 0.935 \\
\hline Surgical & 7(38.9) & $11(61.1)$ & & & \\
\hline
\end{tabular}


The proportion of AKI patients with mortality was higher in those who were 65 years and above (OR: $2.96,95 \%$ CI: $1.06-8.22, \mathrm{p}=0.034)$. There was a significant increase in mortality rate of AKI patients across stages 1 to 3 from $16.7 \%$ to $50 \%$ (OR: 5.0 95\% CI: 0.99-25.08, p=0.046). Mortality was the highest in patients who had AKI from cardiac failure and sepsis compared with other aetiologies, although this was not statistically significant. Mortality rate across the three specialties was not significantly different (Table $3)$.

Age $\geq 65$ years (Adjusted OR: 4.14, CI: $1.32-$ 13.04, $\mathrm{p}=0.015$ ) and AKI stage 3 (Adjusted OR: 6.79, CI: 1.21-38.04, $\mathrm{p}=0.029)$ were still independent predictors of mortality in AKI patients on binary logistic regression (Table 4).

Table 4: Binary logistic regression showing independent predictors of mortality in AKI patients.

\begin{tabular}{llll}
\hline & P value & AOR & 95\% Confidence interval \\
\hline Age 65 years and above & 0.029 & 6.79 & $1.21-38.04$ \\
AKI KDIGO stage 3 & 0.015 & 4.14 & $1.32-13.04$ \\
\hline
\end{tabular}

\section{DISCUSSION}

Acute kidney injury is a common disorder worldwide that is associated with high morbidity, mortality and cost (1, 2, 7-9). The majority of patients with AKI in this study were young and middle aged which is similar to previous reports from tropics (7, 16-18), unlike in developed countries where the elderly are commonly affected $(19,20)$. The more affected population in this study are the economically productive age group of the nation. Hence AKI, contributes to socioeconomic loss in developing countries like Nigeria. There were more males with AKI than females in this study which is similar to previous reports $(21,22)$,although some other authors have reported female predominance in their studies (16, 23). This may be due to the fact that males have better access to health care compared to females in Nigeria as reported by Onah et al (24).

The commonest causes of AKI were sepsis and hypovolaemia. This finding is similar with reports of previous studies $(7,8,16,21,23)$. Medical causes of AKI were also the most predominant when the aetiology were studied across specialties, therefore patients admitted to medical wards should be monitored regularly in order to prevent or diagnose AKI early. The common causes of AKI identified in this study are largely preventable and treatable by regular health education of the public, early diagnosis and adequate treatment by health care workers. Amongst the causes of AKI, heart failure and sepsis had the highest mortality. This is comparable to earlier report by Soliman (25).

More than half of the patients presented late in stage $3 \mathrm{AKI}$, according to KDIGO criteria. Other studies in different parts of Nigeria reported similar findings $(7,23)$. This may be related to poor health care seeking attitude of Nigerians even amongst those who are educated as reported by Chukwuezi et al. (26). Most cases of AKI are treatable and often reversible if managed adequately and promptly (27). Stewart et al reported that only $50 \%$ of patients with AKI received good care in the hospital (28).It was also reported that up to $23.5 \%$ of over 1500 patients admitted in a hospital in Glasgow with AKI were undiagnosed (29). Thus, primary health care givers also need to be regularly educated on how to recognize AKI early and when it becomes expedient for them to refer patients with AKI to nephrologists. Educational intervention was reported to have improved clinicians' knowledge and awareness of acute kidney injury (AKI), which led to better management and outcome in patients with AKI (30).

About $62 \%$ of patients with AKI who needed RRT were dialyzed in this study which is higher compared to $21.4 \%$ reported by Effal et al (23). This may be because the cost of dialysis is relatively cheaper in our centre compared to the latter hospital. Also, AKI patients are treated as emergency in our centre, and those who could not readily afford dialysis immediately are given the opportunity to have one or two sessions of 
dialysis on deferred payment terms. However, the cost of dialysis is still beyond the reach of most Nigerian patients with AKI and places huge financial burden on these patients and their relatives. The majority of these patients stay longer in the hospital after discharge due to inability to settle their hospital bills. It is therefore highly imperative for the government to subsidize renal care in order to reduce overall mortality of patients with AKI.

Ten of our patients who had indication for RRT could not be dialysed due haemodynamic instability from severe hypotension that was refractory to inotropes and fluids. Continuous Renal Replacement Therapy (CRRT), which is the choice of modality of RRT in such critically ill patients, is not available in our centre and in most tertiary health institutions in Nigeria. This mode of therapy is very expensive, and most patients cannot afford it unless it is well subsidized.

The mortality rate of patients with AKI was $38.5 \%$ which is comparable to $39.4 \%$ reported by Bamgboye et al (7) but higher than $18 \%$ reported by Kaballo et al.(31). The lower prevalence in the latter study may be the result of exclusion of major surgical and intensive care unit patients that were included in the index study. However, mortality rate in our study was lower than $46.8 \%$ reported by Emem-Chioma et al. This may be due to the fact that they studied patients with severe AKI who required RRT unlike this study which involved stages 1 to 3 AKI patients (21). The mortality rate is still unacceptably high, and efforts must be intensified in order to achieve ISN vision 0 by 2025 .

Significant factors associated with mortality in this study were older age and late presentation. Elderly patients with AKI were four times more likely to die compared to the young and middle aged patients. This associated increase in mortality in the elderly may be related to the presence of other co-morbidities such as hypertension, diabetes mellitus and heart failure which are common in this age group, although these were not accessed in our study. There was significantly increased mortality across AKI stages 1 to 3 with stage 3 patients having about eight times more risk of mortality compared to AKI stage 1. Similar findings were reported in other studies. (7,21-23) Therefore, early presentation with prompt, adequate care and easy access to all forms of RRT will significantly reduce mortality associated with AKI.

The limitation of this study was that we depended on the accuracy of previous documentations in the patients' case notes and dialysis records because it was a retrospective study. Secondly, the study had a relatively smaller sample size compared to some earlier studies. However, this is the first study in Southwest Nigeria that used the new KDIGO staging of AKI. It also determined the independent predictors of outcomes in AKI patients. The findings in this study will be very useful in advocating for health care policies that will reduce the burden of AKI.

Regular education of the public and health care workers should be done in order to encourage early presentation, prompt diagnosis and treatment. Also, the government should implement policies that will make RRT easily accessible and affordable to those who require it.

In conclusion, sepsis and hypovolaemia were the commonest causes of AKI affecting more of the young and middle aged population. Acute kidney injury-related mortality is still unacceptably high and factors associated with mortality were late presentation and older age.

\section{REFERENCES}

1. Li PK, Burdmann EA, Mehta RL. Acute kidney injury: global health alert. $J$ Nephropathology,2013;2(2):90-97.

2. Susatitaphong P, Cruz DN, Cerda J, Abulfaraj M, Alqahtani F, Koulouridis I et al. World incidence of AKI: a meta-analysis.Clin J Am SocNephrol, 2013;8(9):1482-93.

3. Lameire N, VenBiesenW, Vanholder R. The changing epidemiology of acute renal failure. Nat Clin Pract Nephrol, 2006;2:364-377.

4. Lameire N, VenBiesenW, Vanholder R. Acute renal failure. Lancet 2005;365:417-430

5. Cerda J, Lameire N, Eggers P, Pannu N, Uchino $\mathrm{S}$, Wang $\mathrm{H}$ et al. Epidemiology of acute kidney injury. Clin $J$ Am Nephrol, 2008;3:881-886.

6. Case J, Khan S, Khalid R, Khan A. Epidemiology of acute kidney injury in intensive care unit. Crit Care Res Pract, 2013;4:9,A rticleID479730 http://dx.doi.org/10.1155/2013/47973. 
7. Bamgboye EL, Mabayoje MO, Odutola TA, Mabadeje AF. Acute renal failure at Lagos University Teaching Hospital: a 10 year review. Ren Fail, 1993;15(1):77-80.

8. Okunola OO, Ayodele EO, Adekanle AD. Acute kidney injury requiring haemodialysis in the tropics. Saudi J Kidney DisTranspl, 2012;23(6):1315-9.

9. Hobson C,Ozrazgat-Baslanti T, Kuxhausen A, Thottakkara P, Efron PA, Moore FA et al. Cost and mortality associated with postoperative acute kidney injury. Ann Thorac Surg, 2014;97(1):111-117.

10. Schiffl H, Lang SM, Fischer R. Long term outcomes of survivor of intensive care unit acute kidney injury requiring renal replacement therapy: a 10 year prospective cohort study. Clin Kidney J 2012;5:297-302.

11. Coca SG, Singanamala S, Parikh CR. Chronic kidney disease after acute kidney injury: a systematic review and metanalysis. Kidney Int, 2012;81:442-448.

12. Abefe SA, Abiola AF, Olubunmi AA, Adewale A. Utility of predicted creatinine clearance using MDRD formula compared with other predictive formulas in Nigerian patients. Saudi J Kidney Dis Transpl, 2009; 20(1):86-90.

13. KDIGO Clinical Practice for Acute Kidney Injury. Kidney Int, 2012;2(Suppl1):1-141.

14. Macedo E, Bouchard J, Mehta RL. Renal recovery following acute kidney injury. Curr Opin Crit Care, 2008; 14:660-665.

15. Levy MM, Fink MP, Marshall JC, Abraham E, AngusD,CookD.2001SCCM/ESICM/ACCO/ ATS/SIS. International Sepsis Definitions Conference. Crit Care Med, 2003;31(4):12501256.

16. Chijioke A. The pattern of Acute renal failure in Ilorin, Nigeria. Orient Journal of Medicine 2003;15(1):18-23.

17. Kohli HS, Bhat A, Jairam A, Aravindan AN, Sud K, Jha V, Gupta KL, Sakhuja V: Predictors of mortality in acute renal failure in a developing country: A prospective study. Ren Fail, 2007;29 :463- 469.

18. Kaul A, Sharma RK, Tripathi R, Suresh KJ, Bhatt S, Prasad N. Spectrum of communityacquired acute kidney injury in India: a retrospective study. Saudi J Kidney Dis Transpl, 2012;23(3):619-28.
19. Xue JL, Daniels F,Star RA, Kimmel PL, Eggers PW, Molitoris BA, Himmelfarb J, Collins AJ: Incidence and mortality of acute renal failure in Medicare beneficiaries, 1992 to 2001. J Am Soc Nephrol, 2006:1135-1142.

20. Waikar SS, Curhan GC, Wald R, McCarthy EP, Chertow GM: Declining mortality in patients with acute renal failure, 1988 to 2002. J Am Soc Nephro, 2006:17:1143-1150

21. Emem-Chioma PC, Alasia DD, Wokoma FS. Clinical outcomes of dialysis treated Acute kidney Injury patients at University of PortHarcourt Teaching Hospital, Nigeria. ISRN Nephrol, 2013;3:6. doi:10.5402/2013/540526.

22. Bagasha P, Nakwagala F, Kwizera A, Ssekasanvu E, Kalysesubula R. Acute Kidney Injury among adult patients with sepsis in a low-income country: Clinical pattern and short term outcomes. BMC Nephrology, 2015;16(4):7. doi:10.1186/1471-2369-16-4.

23. Effal EE, Okpa HO, Mbu PN, Epoke EJ, Otokpa DE. Acute Kidney Injury in hospitalized patients at University of Calabar Teaching Hospital; An aetiological and outcome study. JDMS, 2015;14(3):55-59.

24. Onah MN, Govender V. Out-of-pocket payments, health care accesss and utilization in south-eastern Nigeria: A gender perspective. PLos ONE, 2014;9(4):e93887. doi:10.1371/journal.pone.0093887 accessed on the $4^{\text {th }}$ September 2015.

25. Soliman AR. Spectrum of Acute Kidney Injury in a tertiary hospital in Cairo. AJNT, 2011;4(2):83-86.

26. Chukwuezi CO, Anelechi AB. Factors associated with delay in seeking medical care among educated Nigerians. Asian Journal of Medical Science, 2009;1(2):30-32.

27. Rewa O, Bagshaw SM. Acute kidney injuryepidemiology, outcomes and economics. Nat Rev Nephrol, 2014;10:193-207.

28. Stewart JA. Adding insult to injury: care of patients with acute kidney injury. $\mathrm{Br} \quad J$ HospMed (Lond) 2009;70(7):372-3.

29. Aitken E, Carruthers C, Gall L, Kerr L, Geddes C, Kingsmore D. Acute kidney injury: outcomes and quality of care. $Q J M$, 2013;106(4):323-332.

30. Xu G, BainesR,Westacott R, Selby N, Carr S An educational approach to improve outcomes in acute kidney injury (AKI): report of a 
quality improvement project. BMJ Open, 2014;4:e004388 doi:10.1136/bmjopen-2013004388 accessed on $13^{\text {th }}$ August, 2015.

31. Kaballo GB, Khogali MS, Khalifa EH, Khalil EAG, El-Hassan AM, Abu-Aisha H. Pattern of
"Severe" Acute Renal Failure in a referral centre in Sudan: excluding intensive care and major surgical patients. Saudi J Kidney DisTranspl, 2007;18(2):220-5. 